\title{
Special Issue: Harnessing theories for tool support in software
}

\author{
Volker Stolz
}

Received: 5 November 2012 / Accepted: 9 November 2012 / Published online: 22 November 2012

(C) Springer-Verlag London 2012

This issue contains selected contributions from the International Workshop on Harnessing Theories for Tool Support in Software (TTSS) series. Based on their past contributions, participants were invited to submit extended versions of their research. The workshop brings together practitioners and researchers from academia and industry to present and discuss ideas about:

- How to deal with the complexity of software projects by multi-view modeling and separation of concerns about the design of functionality, interaction, concurrency, scheduling, and non-functional requirements.

- How to insure correctness and dependability of software by integrating formal methods and tools for modeling, design, verification, and validation into design and development processes and environments.

- Case studies and experience that report about harnessing static analysis tools such as model checking, theorem proving, testing, and runtime monitoring.

The workshop was initiated under the auspices of Prof. Jifeng He (ECNU, China) and Dr. Zhiming Liu (UNU-IIST, Macao). Also, Patrick Cousot (ENS, France), Mathai Joseph (TATA, India), Bertrand Meyer (ETH Zurich, Switzerland), and Jim Woodcock (U. York, UK) kindly agreed to lend their advice. The past workshops have been held at:

V. Stolz

Department of Informatics, University of Oslo, Oslo, Norway

V. Stolz $(\bowtie)$

United Nations University-International Institute for Software

Technology (UNU-IIST), Macao SAR, China

e-mail: vs@iist.unu.edu; stolz@ifi.uio.no
- UNU-IIST, 22-23 September 2007, Macao SAR, China

- Sabancı University, 30 August 2008, Istanbul, Turkey

- Universiti Kebangsaan Malaysia, 17 August 2009, Kuala Lumpur, Malaysia

- East China Normal University, 15 November 2010, Shanghai, China

For this special issue, the following papers were accepted after careful reviewing for their interest to the community: Griesmayer et al. combined in "A Framework for Automated and Certified Refinement Steps" semi-automated refinement steps for the object-oriented part of the rCOS language with their correctness proofs. The rewriting tool Maude is used to search for possible refinements, and upon application, proof obligations for the Isabelle theorem prover are generated and attempted to discharge. In "Towards Harnessing Theories through Tool Support for Hard Real-Time Java Programming", Rydhof Hansen et al. motivate their choice for the selection of tools for the development of hard real-time applications on the Java platform. An abstract treatment of realtime concerns is given by Bjørk et al. where "User-defined Schedulers for Real-Time Concurrent Objects" are presented as an expressive means in the high-level object-oriented modeling language Real-Time ABS to specify, simulate and analyze application-specific schedulers. In "Model Checking Driven Static Analysis for the Real World", Fehnker and Huuck describe the challenges while developing a commercial static analysis tool (based on model checking techniques) for $\mathrm{C} / \mathrm{C}++$. "Modeling and Analysis of Interactive Telemedicine Systems" by Liu Jing et al. addresses the timely issue of development of reliable software.

The workshops could not have been held without the help of others; my thanks go to the co-chairs Min Zhang and Geguang $\mathrm{Pu}$ at the East China Normal University in Shanghai, China, and Einar Broch Johnsen at the University 
of Oslo, Norway. Additional thanks go out to the members of the program committees and the additional reviewers for this special issue.
Acknowledgments Editorial activity partially supported by the ARV Grant of the Macao Science and Technology Development Fund. 